\title{
Compendium of Current Single Event Effects Results for Candidate Spacecraft Electronics for NASA
}

\author{
Martha V. O'Bryan, Christian Poivey, Kenneth A. LaBel, Stephen P. Buchner, Ray L. Ladbury, \\ Timothy R. Oldham, James W. Howard, Jr., Anthony Sanders, Melanie Berg and Jeffrey L. Titus
}

\begin{abstract}
Sensitivity of a variety of candidate spacecraft electronics to proton and heavy ion induced single event effects is presented. Devices tested include digital, linear, and hybrid devices.
\end{abstract}

Index Terms-Single Event Effects, spacecraft electronics, digital, linear bipolar, and hybrid devices.

\section{INTRODUCTION}

In order to meet the demands of reduced cost, higher performance and more rapid delivery schedules imposed by the space flight community, commercial and emerging technology devices have assumed a prominent role in meeting these needs. The importance of ground-based testing of such devices for susceptibility to single event effects (SEE) has assumed greater importance. The novel ways in which some of these devices are used also highlights the need for application specific testing to ensure their proper operation and ability to meet mission goals.

This work was supported in part by the NASA Electronic Parts and Packaging Program (NEPP), NASA Flight Projects, and the Defense Threat Reduction Agency (DTRA).

Martha V. O'Bryan, MEI Technologies Inc., c/o NASA Goddard Space Flight Center (GSFC), Code 561.4, Bldg. 22, Rm. 062A, Greenbelt, MD 20771 (USA), phone: 301-286-1412, fax: 301-286-4699, email: martha.obryan@gsfc.nasa.gov.

Christian Poivey, and Melanie Berg, MEI Technologies, Inc., c/o NASA/GSFC, Code 561.4, Greenbelt, MD 20771 (USA), phone: 301-2862128 (Poivey), 301-286-2153 (Berg), email: cpoivey@pop500 .gsfc.nasa.gov, and Melanie.D.Berg.1@gsfc.nasa.gov.

Kenneth A. LaBel, Ray L. Ladbury and Anthony B. Sanders, NASA/GSFC, Code 561.4, Greenbelt, MD 20771 (USA), phone: 301-2869936 (LaBel), 301-286-1030 (Ladbury), 301-286-1151 (Sanders), email: Kenneth.A.Label@ nasa.gov, Raymond.L.Ladbury@nasa.gov and Anthony.B.Sanders@nasa.gov.

Stephen P. Buchner, and Timothy R. Oldham, Perot Systems, c/o NASA/GSFC, Code 561.4, Greenbelt, MD 20771 (USA), phone: 301-2865019 (Buchner), 301-286-5489 (Oldham), email: sbuchner@pop500. gsfc.nasa.gov, and timothy.r.oldham@gsfc.nasa.gov.

James W. Howard, Jr., Jacobs Engineering (formerly MEI Technologies, Inc.), Huntsville AL 35806.

Jeffrey L. Titus, NAVSEA - Crane Radiation Sciences Branch, phone: 812-584-1617, email: jeffrey.titus@navy.mil.
The studies discussed here were undertaken to establish the sensitivities of candidate spacecraft electronics to heavy ion and proton-induced single event upset (SEU), single event latchup (SEL), and single event transient (SET). For proton displacement damage (DD) and total ionizing dose (TID) results, see a companion paper submitted to the 2007 IEEE NSREC Radiation Effects Data Workshop entitled: "Compendium of Current Total Ionizing Dose Results and Displacement Damage Results for Candidate Spacecraft Electronics for NASA" by D. Cochran, et al. [1].

\section{TEST TECHNIQUES AND SETUP}

\section{A. Test Facilities}

All SEE tests were performed between February 2006 and February 2007. Heavy ion experiments were conducted at Lawrence Berkeley National Laboratory (LBNL) [2], at Texas A\&M University Cyclotron (TAMU) [3], and at the Single Event Effects Test Facility (SEETF) at the National Superconducting Cyclotron Laboratory (NSCL) at Michigan State University (MSU) [4]. The LBNL and TAMU facilities use an 88" cyclotron. The NSCL MSU facility uses tandem K500 and K1200 cyclotrons to deliver on target ions with energies up to $125 \mathrm{MeV} / \mathrm{n}$. All these facilities are suitable for providing a variety of ions over a range of energies for testing. The DUT was irradiated with heavy ions having linear energy transfers (LETs) ranging from 0.59 to $120 \mathrm{MeV} \cdot \mathrm{cm}^{2} / \mathrm{mg}$. Fluxes ranged from $1 \times 10^{3}$ to $1 \times 10^{7}$ particles $/ \mathrm{cm}^{2}$ per second, depending on the device sensitivity. Representative ions used are listed in Table I. LETs between the values listed were obtained by changing the angle of incidence of the ion beam with respect to the DUT, thus changing the path length of the ion through the DUT and the "effective LET" of the ion [5]. Energies and LETs available varied slightly from one test date to another.

Proton SEE tests were performed at two facilities: the University of California at Davis (UCD) Crocker Nuclear Laboratory (CNL) [6], and at the Indiana University Cyclotron Facility (IUCF) [7]. Proton test energies incident on the DUT are listed in Table II. Proton SEE tests were performed in a manner similar to heavy ion exposures. However, because protons cause SEE via indirect ionization of recoil particles, results are parameterized in terms of proton energy rather than LET. Because such proton-induced nuclear interactions are rare, proton tests also feature higher cumulative fluence and particle flux rates than do heavy ion experiments. 
TABLE I: HEAVY ION TEST FACILITIES AND TEST HEAVy IONS

\begin{tabular}{|c|c|c|c|c|}
\hline & Ion & $\begin{array}{c}\text { Energy } \\
(\mathrm{MeV})\end{array}$ & $\begin{array}{c}\text { Surface } \\
\mathrm{LET} \text { in } \mathrm{Si} \text {, } \\
\mathrm{MeV} \cdot \mathrm{cm}^{2} / \mathrm{mg} \\
\text { (Normal } \\
\text { Incidence) }\end{array}$ & $\begin{array}{c}\text { Range in } \\
\text { Si }(\mu \mathrm{m})\end{array}$ \\
\hline \multirow[t]{7}{*}{ LBNL } & $\mathrm{O}^{18}$ & 184 & 2.2 & 227 \\
\hline & $\mathrm{Ne}^{22}$ & 216 & 3.5 & 175 \\
\hline & $\mathrm{Ar}^{40}$ & 400 & 9.7 & 130 \\
\hline & $\mathrm{Cu}^{65}$ & 659 & 21 & 110 \\
\hline & $\mathrm{Kr}^{86}$ & 886 & 31 & 110 \\
\hline & $X e^{136}$ & 1330 & 59 & 97 \\
\hline & \multicolumn{4}{|c|}{$10 \mathrm{MeV}$ per AMU tune } \\
\hline MSU & $X e^{124}$ & 17360 & 14.1 & $\sim 3300$ \\
\hline \multirow[t]{9}{*}{ TAMU } & $\mathrm{Ne}^{20}$ & 300 & 2.5 & 316 \\
\hline & $\mathrm{Ar}^{40}$ & 599 & 7.7 & 229 \\
\hline & $\mathrm{Cu}^{63}$ & 944 & 17.8 & 172 \\
\hline & $\mathrm{Kr}^{84}$ & 1259 & 25.4 & 170 \\
\hline & $\mathrm{Xe}^{29}$ & 1934 & 47.3 & 156 \\
\hline & \multicolumn{4}{|c|}{$15 \mathrm{MeV}$ per AMU tune } \\
\hline & $\mathrm{Ne}^{20}$ & 800 & 1.2 & 1655 \\
\hline & $\mathrm{Ar}^{40}$ & 1598 & 3.8 & 1079 \\
\hline & \multicolumn{4}{|c|}{$40 \mathrm{MeV}$ per AMU tune } \\
\hline
\end{tabular}

TABLE II: PROTON TEST FACILITIES

University of California at Davis (UCD) Crocker Nuclear Laboratory (CNL), energy $63 \mathrm{MeV}$, flux ranged from $8 \times 10^{7}$ to $1 \times 10^{9}$ particles $/ \mathrm{cm}^{2} / \mathrm{s}$.

Indiana University Cyclotron Facility (IUCF), energy ranged from 50 to $200 \mathrm{MeV}$; flux ranged from $1 \times 10^{7}$ to $8 \times 10^{8}$ particles $/ \mathrm{cm}^{2} / \mathrm{s}$.

\section{B. Test Method}

Unless otherwise noted, all tests were performed at room temperature and with nominal power supply voltages. We recognize that high-temperature and worst-case power supply conditions are recommended for single event latchup (SEL) device qualification.

\section{1) SEE Testing - Heavy Ion:}

Depending on the DUT and the test objectives, one or more of three SEE test methods were typically used:

Dynamic - the DUT was exercised continually while being exposed to the beam. The events and/or bit errors were counted, generally by comparing DUT output to an unirradiated reference device or other expected output (Golden chip or virtual Golden chip methods). In some cases, the effects of clock speed or device operating modes were investigated. Results of such tests should be applied with caution due to the application-specific nature of the results.

Static - the DUT was loaded prior to irradiation; data were retrieved and errors were counted after irradiation.

Biased - the DUT was biased and clocked while $\mathrm{I}_{\mathrm{CC}}$ (power consumption) was monitored for SEL or other destructive effects. In most SEL tests, functionality was also monitored.

In SEE experiments, DUTs were monitored for soft errors, such as SEUs and for hard errors, such as SEL. Detailed descriptions of the types of errors observed are noted in the individual test results. [8]

SET testing was performed using a high-speed oscilloscope. Individual criteria for SETs are specific to the device being tested. Please see the individual test reports for details. [8]

Heavy ion SEE sensitivity experiments include measurement of the Linear Energy Transfer threshold $\left(\mathrm{LET}_{\text {th }}\right)$ and saturation cross section at maximum measured LET. The $\mathrm{LET}_{\text {th }}$ is defined as the maximum LET value at which no effect was observed at an effective fluence of $1 \times 10^{7}$ particles $/ \mathrm{cm}^{2}$. In the case where events are observed at lower fluences for the smallest LET tested, $\mathrm{LET}_{\text {th }}$ will either be reported as less than the lowest measured LET or determined approximately as the $\mathrm{LET}_{\text {th }}$ parameter from a Weibull fit.

2) SEE Testing - Proton

Proton SEE tests were performed in a manner similar to heavy ion exposures. However, because protons cause SEE via indirect ionization of recoil particles, results are parameterized in terms of proton energy rather than LET. Because such proton-induced nuclear interactions are rare, proton tests also feature higher cumulative fluences and particle flux rates than do heavy ion experiments.

\section{TEST RESUlts OVERVIEW}

Abbreviations and conventions are listed in Table III. Abbreviations for principal investigators (PIs) are listed in Table IV, and SEE results are summarized in Table V. Unless otherwise noted, all LETs are in $\mathrm{MeV} \cdot \mathrm{cm}^{2} / \mathrm{mg}$ and all cross sections are in $\mathrm{cm}^{2} /$ device. This paper is a summary of results. Complete test reports are available online at http://radhome.gsfc.nasa.gov [8]. 
TABLE III: ABBREVIATIONS AND CONVENTIONS:

$$
\mathrm{H}=\text { heavy ion test }
$$

$\mathrm{P}=$ proton test $(\mathrm{SEE})$

Samp = sample

P.I. = principal investigator

$\mathrm{LDC}=$ lot date code

DUT $=$ device under test

LET $=$ linear energy transfer $\left(\mathrm{MeV} \cdot \mathrm{cm}^{2} / \mathrm{mg}\right)$

$\mathrm{LET}_{\text {th }}=$ linear energy transfer threshold (the minimum LET value for which a given effect is observed for a fluence of $1 \times 10$ particles $/ \mathrm{cm}^{2}$ - in $\mathrm{MeV} \cdot \mathrm{cm}^{2} / \mathrm{mg}$ )

$<=$ SEE observed at lowest tested LET

$>=$ No SEE observed at highest tested LET

$\sigma=$ cross section $\left(\mathrm{cm}^{2} /\right.$ device, unless specified as $\mathrm{cm}^{2} /$ bit $)$

$\sigma_{\text {max measured }}=$ cross section at maximum measured LET $\left(\mathrm{cm}^{2} /\right.$ device, unless specified as $\left.\mathrm{cm}^{2} / \mathrm{bit}\right)$

App. Spec. $=$ application specific

Aux = auxiliary

CMOS = complementary metal oxide semiconductor

$\mathrm{DDR}=$ double data rate

FPGA = field programmable gate array

$\mathrm{V} / \mathrm{O}=$ input/output

$\mathrm{LVDO}=$ low voltage drop out

$\mathrm{MMIC}=$ monolithic microwave integrated circuit

MOSFET $=$ metal oxide semiconductor field effect transistor

$\mathrm{N} / \mathrm{A}=$ not applicably

NAND $=$ not and (electronic logic gate)

Op Amp = operational amplifier

PAL = programmable array logic

$\mathrm{pHEMT}=\mathrm{p}$-type high electron mobility transistor

$\mathrm{PPC}=$ power $\mathrm{PC}$

SDRAM = synchronous dynamic random access memory

SEBE $=$ single event burst error

$\mathrm{SEE}=$ single event effect

SEFI = single event functional interrupt

SEGR = single event gate rupture

$\mathrm{SEL}=$ single event latchup

$\mathrm{SET}=$ single event transient

SEU $=$ single event upset

VIN or VOUT $=$ input voltage or output voltage
TABLE IV: LIST OF PRINCIPAL INVESTIGATORS

\begin{tabular}{|l|c|}
\hline \multicolumn{1}{|c|}{ Principal Investigator (PI) } & Abbreviation \\
\hline Steve Buchner & SB \\
\hline Melanie Berg & $\mathrm{MB}$ \\
\hline Jim Howard & $\mathrm{JH}$ \\
\hline Ray Ladbury & $\mathrm{RL}$ \\
\hline Timothy Oldham & $\mathrm{TO}$ \\
\hline Christian Poivey & $\mathrm{CP}$ \\
\hline Anthony (Tony) Sanders & $\mathrm{TS}$ \\
\hline
\end{tabular}


TABLE V: SUMMARY OF SEE TEST RESULTS

\begin{tabular}{|c|c|c|c|c|c|c|c|c|c|c|}
\hline $\begin{array}{l}\text { Part } \\
\text { Number }\end{array}$ & Manufacturer & LDC & $\begin{array}{l}\text { Technologyl } \\
\text { Device } \\
\text { Function }\end{array}$ & Process & $\begin{array}{c}\text { Particle: } \\
\text { (Facility/Date) P.I., }\end{array}$ & $\begin{array}{c}\text { Test Results } \\
\text { LET in } \mathrm{MeV} \cdot \mathrm{cm}^{2} / \mathrm{mg} \\
\sigma \text { in } \mathrm{cm}^{2} / \text { device, unless } \\
\text { otherwise specified } \\
\end{array}$ & $\begin{array}{l}\text { App. } \\
\text { Spec. } \\
\text { Test } \\
\text { (Y/N) } \\
\end{array}$ & $\begin{array}{l}\text { Supply } \\
\text { Voltage }\end{array}$ & $\begin{array}{l}\text { Samp. } \\
\text { Size }\end{array}$ & Test Report \\
\hline \multicolumn{11}{|c|}{ Programmable LogiciFPGA } \\
\hline Eclipse FPGA & Aeroflex & $\begin{array}{c}1059 \text { and } \\
1082\end{array}$ & $\begin{array}{l}0.25 \mu \mathrm{m} \text { CMOS } \\
\text { shift register }\end{array}$ & CMOS & $\begin{array}{l}\text { H: (TAMU06MAY) MB; } \\
\text { H: (TAMUO6NOV) MB; } \\
\text { H: (LBNLO7JAN) MB; } \\
\text { P: (IUOGMAR) MB }\end{array}$ & $\begin{array}{l}\text { H: } 8.5<\text { SEU LET } \text { th }_{\text {th }}<12 ; \\
\text { P: SEU LET } \\
195 \mathrm{MeV} \text { Protons. No errors } \\
\text { observed at lower energies. }\end{array}$ & $\mathrm{N}$ & $\begin{array}{c}\text { Core 2.5V; } \\
\text { I/O } 3.3 \mathrm{~V}\end{array}$ & 10 & T022205_Aeroflex_Eclipse_v2 \\
\hline RTAX-S & Actel & $\begin{array}{c}0506 \text { and } \\
0543\end{array}$ & $\begin{array}{l}0.15 \mu \mathrm{m} \text { CMOS } \\
\text { FPGA }\end{array}$ & CMOS & $\begin{array}{l}\text { H: (TAMUOGMAY) MB; } \\
\text { P: (IUOGMAR) MB }\end{array}$ & \begin{tabular}{|l|}
$\mathrm{H}$ : SEU LET \\
if running at a slower frequency); \\
P: SEU $\sigma \sim 8.5 \times 10^{-16}$ with 195 \\
MeV; $6.6 \times 10^{-16}$ with $63 \mathrm{MeV}$ \\
Protons at $150 \mathrm{MHz}$; \\
No SEUs observed at $15 \mathrm{MHz}$ at \\
both 63 and $195 \mathrm{MeV}$ Protons. \\
\end{tabular} & $\mathrm{N}$ & $\begin{array}{c}\text { Core: } \\
1.5 \mathrm{~V} \\
\mathrm{I} / \mathrm{O}: 3.3 \mathrm{~V}\end{array}$ & 10 & T110405_RTAX_v2 \\
\hline LX25 & Xilinx & 0553 & $\begin{array}{l}\text { Virtex IV FPGA } \\
90 \mathrm{~nm} \text { CMOS } \\
\text { flip chip }\end{array}$ & CMOS & \begin{tabular}{|l|} 
H: (TAMUO6MAY) MB; \\
H: (TAMUO6JUN) MB; \\
H: (TAMUO6AUG) MB; \\
P: (IUO6MAR) MB; \\
P: (IUO6JUL) MB \\
\end{tabular} & $\begin{array}{l}\text { H: SEL LET } T_{\text {th }}>75 ; \\
\text { SEFI LETth<5.7; } \\
\text { P: No SEL observed at both } 93 \\
\text { and } 195 \text { MeV Protons. }\end{array}$ & $\mathrm{N}$ & $\begin{array}{c}\text { Core } 1.2 \mathrm{~V} \\
\mathrm{I} / \mathrm{O} 3.3 \mathrm{~V}\end{array}$ & 1 & [1032706_LX25 \\
\hline XC4VFX60 & Xilin $x$ & $\begin{array}{c}\text { 0629(MSU), } \\
\text { 0609(TAMU) }\end{array}$ & $\begin{array}{l}\text { Virtex IV FPGA } \\
90 \mu \mathrm{m} \text { CMOS }\end{array}$ & CMOS & $\begin{array}{l}\text { H: (MSU06OCT) CP; } \\
\text { H: (TAMU07FEB) CP }\end{array}$ & $\begin{array}{l}\text { SEL LET }>\text { th }>58 ; \text { SEFI LET } \text { Lth }_{\text {th }}<5.7 \\
\text { SEFI } \sigma_{\mathrm{SAT}}=1 \times 10^{-2} \text { with embedded } \\
\text { PPC. }\end{array}$ & $\mathrm{Y}$ & $\begin{array}{l}\text { Core } 12 \mathrm{~V} \\
\text { I/O } 3.3 \mathrm{~V} \\
\text { Aux } 2.5 \mathrm{~V}\end{array}$ & $\begin{array}{l}2 \text { (MSU), } \\
\text { 3(TAMU) }\end{array}$ & $\begin{array}{l}\text { M102606_XC4VFX60, and } \\
\text { T021607_XC4VFX60 }\end{array}$ \\
\hline AT22V10 & Cypress & 0437A & PAL & $\begin{array}{c}1 \mathrm{~nm} \\
\text { BiCMOS }\end{array}$ & H: (TAMU06AUG) CP & $\begin{array}{l}1 \text { failure at LET 106; } \\
2 \text { failures at LET } 53 \\
\text { SEU LET } \\
\text { th }<2.8 ; \text { SEU } \sigma>1 \times 10^{-3}\end{array}$ & $\mathrm{~N}$ & $5 \mathrm{~V}$ & 4 & T082606_AT22V10 \\
\hline \multicolumn{11}{|c|}{ Linear Devices } \\
\hline $\mathrm{RH} 137 \mathrm{H}$ & $\begin{array}{l}\text { Linear } \\
\text { Technology }\end{array}$ & 0235 & $\begin{array}{l}\text { Negative } \\
\text { Adjustable } \\
\text { Regulator }\end{array}$ & Bipolar & H: (TAMU06MAY) CP & \begin{tabular}{|l|} 
SET LET \\
output capacitor; $>$ SET LET LT $_{\text {th }} \sim 20$ \\
without output capacitor; Positive \\
and negative transients observed; \\
SET $\sigma>3 \times 10^{-3}$
\end{tabular} & Y & \begin{tabular}{|c|} 
Vin -15 \\
Vout $-12 \mathrm{~V}$
\end{tabular} & 2 & T052306_RH137 \\
\hline TPS76701 & \begin{tabular}{|l|} 
Texas \\
Instruments
\end{tabular} & \begin{tabular}{c|} 
LDC \\
unknown - \\
no package \\
markings
\end{tabular} & \begin{tabular}{|l} 
Voltage \\
Regulator
\end{tabular} & BiCMOS & H: (LBNLO6JUN) CP & $\begin{array}{l}\text { SEL LET } \\
\text { th }>83 \\
\text { SET LET } \\
\text { SET } \sigma \sim 2 \times 10^{-3}<3\end{array}$ & $\mathrm{~N}$ & \begin{tabular}{|c|} 
Vin $3.3 ;$ \\
Vout $1.5 \mathrm{~V}$
\end{tabular} & 3 & L061606_TPS76701, [13] \\
\hline TPS73601 & \begin{tabular}{|l|} 
Texas \\
Instruments
\end{tabular} & $\begin{array}{l}\text { LDC not } \\
\text { indicated }\end{array}$ & $\begin{array}{l}\text { Low dropout } \\
\text { linear } \\
\text { adjustable } \\
\text { voltage } \\
\text { regulator } \\
\end{array}$ & BiCMOS & H: (LBNL06JUN) CP & $\begin{array}{l}\text { SEL LETth }>59 ; \text { SET LET } \text { th }<2 \text { at } \\
\text { highest load current }\left(I_{\text {out }}=250 \mathrm{~mA}\right) \\
\text { SET sensitivity increased with } \\
\text { load current; } \\
\text { SET } \sigma \sim 1 \times 10^{-3} \text { (worst case) }\end{array}$ & $\mathrm{N}$ & $\begin{array}{c}\text { Vin } 3.3 ; \\
\text { Vout } 1.5 \mathrm{~V}\end{array}$ & 2 & L061806_TPS73601, [13] \\
\hline SAT8605R & $\begin{array}{l}\text { SatCon } \\
\text { Electronics }\end{array}$ & \begin{tabular}{|c|} 
LDC \\
unknown - \\
no package \\
markings
\end{tabular} & $\begin{array}{l}2.5 \text { linear } \\
\text { voltage } \\
\text { regulator }\end{array}$ & Hybrid & H: (LBNL06JUN) CP & $\begin{array}{l}\text { SEL LET } \text { th }_{\text {th }} 59 ; \text { SET LET }_{\text {th }}<21 ; \\
\text { SET } \sigma \sim 8 \times 10^{-4}\end{array}$ & $\mathrm{~N}$ & \begin{tabular}{|c|} 
Vin $3.3 ;$ \\
Vout $1.5 \mathrm{~V}$
\end{tabular} & 2 & L061606_SAT8605R, [13] \\
\hline RH1013 & $\begin{array}{l}\text { Linear } \\
\text { Technology }\end{array}$ & 0343A & Op Amp & Bipolar & H: (TAMUOGNOV) CP & $\begin{array}{l}\text { SEU LET } \text { th }_{\text {tn }} 76 ; \text { SET LET } \\
\text { SET } \sigma \sim 10 ;\end{array}$ & $\mathrm{Y}$ & $+/-15 \mathrm{~V}$ & 2 & T111606_RH1013 \\
\hline MAX997 & Maxim & 0531 & $\begin{array}{l}\text { Voltage } \\
\text { Comparator }\end{array}$ & Bipolar & H: (TAMUOGNOV) AS & $\begin{array}{l}\text { SEL LET } \text { Lth }_{\text {th }}>76 ; \\
\text { SEU LET }\end{array}$ & $\mathrm{Y}$ & $\begin{array}{c}4.3 \mathrm{~V} \text { and } \\
5 \mathrm{~V}\end{array}$ & 2 & T111906_MAX997ESA \\
\hline RHFL4913 & \begin{tabular}{|l|} 
ST \\
Microelectronics
\end{tabular} & 0510A & $\begin{array}{l}\text { Bipolar LVDO } \\
\text { Regulator }\end{array}$ & Bipolar & H: (TAMUO6NOV) AS & 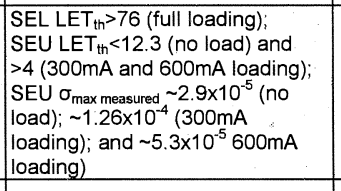 & $\mathrm{Y}$ & $\begin{array}{c}3.3 \mathrm{~V} \text { and } \\
5 \mathrm{~V}\end{array}$ & 2 & T111806_RHFL4913 \\
\hline AСТ8601 & Aeroflex & $\begin{array}{l}\text { No LDC } \\
\text { (hybrid) }\end{array}$ & $\begin{array}{l}\text { Dual voltage } \\
\text { regulator } \\
\mathrm{RH} 117 \text { and } \\
\mathrm{RH} 137\end{array}$ & Bipolar & H: (LBNL06JUN) SB & $\begin{array}{l}\text { SEL LET } \\
\text { SET LET }>59 ; \\
\text { th }>59\end{array}$ & $\mathrm{Y}$ & $\begin{array}{c}+/-12 \mathrm{~V} \\
\text { inputs; }+1- \\
9 \mathrm{~V} \text { outputs }\end{array}$ & 1 & L061806_ACT8601 \\
\hline TL431 & \begin{tabular}{|l} 
Texas \\
Instruments
\end{tabular} & $\begin{array}{l}\text { No LDC; } \\
\text { die pkg at } \\
\text { GSFC }\end{array}$ & $\begin{array}{l}\text { Programmable } \\
\text { Shunt } \\
\text { Regulator }\end{array}$ & Bipolar & H: (LBNLO6JUN) SB & $\begin{array}{l}\text { SEL LET } \\
\text { SET LET }>59 ; \\
\text { SET } \sigma_{\text {max }}<3.3 \\
\text { measured }\end{array}$ & Y & $3.67 \mathrm{~V}$ & 2 & L061806_TL431 \\
\hline LMH6702 & $\begin{array}{l}\text { National } \\
\text { Semiconductor }\end{array}$ & $\begin{array}{l}\text { No LDC Info } \\
\text { on package }\end{array}$ & $\mid \begin{array}{l}\text { High Speed Op } \\
\text { Amp }\end{array}$ & Bipolar & H: (LBNLOGJUN) SB & \begin{tabular}{|l} 
SEL LET \\
SET LET $>81$ \\
SET $\sigma_{\text {max measured }} \sim 10 ; 10^{-6}$ at LET 81
\end{tabular} & Y & $3.8 \mathrm{~V}$ & 1 & L061806_LMH6702 \\
\hline RH1499 & \begin{tabular}{|l} 
Linear \\
Technology
\end{tabular} & 0220 & Op Amp & Bipolar & H: (LBNLO6JUN) SB & $\begin{array}{l}\text { SEL LET } \text { th }_{\text {th }}>58.7 \\
\text { SET LET } \\
\text { SET } \sigma_{\text {SAT }}<3 \text {; } \\
\sim 410^{-3} \text { at LET } 58.7\end{array}$ & $\mathrm{~N}$ & $+/-15 \mathrm{~V}$ & 2 & L061806_RH1499 \\
\hline AD549 & Analog Devices & 0535 & $\begin{array}{l}\text { Ultralow Input- } \\
\text { Bias Current } \\
\text { Operational } \\
\text { Amplifier }\end{array}$ & Bipolar & $\mathrm{H}:$ (LBNL06SEP) AS & $\begin{array}{l}\text { SEL LET } \\
\text { SET LET }>83 ; \\
\text { th }<58.7\end{array}$ & Y & $15 \mathrm{~V}$ & 3 & L091906_AD549 \\
\hline
\end{tabular}


TABLE V: SUMMARY OF SEE TEST RESUlTS (CONT.)

\begin{tabular}{|c|c|c|c|c|c|c|c|c|c|c|}
\hline $\begin{array}{l}\text { Part } \\
\text { Number }\end{array}$ & Manufacturer & LDC & $\begin{array}{l}\text { Technologyl } \\
\text { Device } \\
\text { Function }\end{array}$ & Process & $\begin{array}{l}\text { Particle: (Facility) } \\
\text { P.I., }\end{array}$ & $\begin{array}{c}\text { Test Results } \\
\text { LET in MeV.cm }{ }^{2} / \mathrm{mg} \\
\sigma \text { in } \mathrm{cm}^{2} / \text { device, unless } \\
\text { otherwise specified }\end{array}$ & $\begin{array}{l}\text { App. } \\
\text { Spec. } \\
\text { Test } \\
\text { (Y/N) }\end{array}$ & $\begin{array}{l}\text { Supply } \\
\text { Voltage }\end{array}$ & $\begin{array}{l}\text { Samp. } \\
\text { Size }\end{array}$ & Test Report \\
\hline \multicolumn{11}{|l|}{ Optocouplers } \\
\hline HCPL625K & $\begin{array}{l}\text { Agilent } \\
\text { Technologies }\end{array}$ & 0534 & $\begin{array}{l}\text { Optocoupler; } 4 \\
\text { channels logic } \\
\text { gate }\end{array}$ & $\begin{array}{l}\text { AlGaAs } \\
\text { LED }\end{array}$ & P: (IUOGMAR) CP & $\begin{array}{l}\text { SET } \sigma=1 \times 10^{-7} \mathrm{~cm}^{2} / \text { channel; } \\
\text { Proton energy threshold }<100\end{array}$ & $\mathrm{Y}$ & $5 \mathrm{~V}$ & 4 & 1032706_HCPL625K \\
\hline HCPL-6751 & $\begin{array}{l}\text { Agilent } \\
\text { Technologies }\end{array}$ & 0251 & $\begin{array}{l}\text { Power } \\
\text { MOSFET } \\
\text { Optocouplers }\end{array}$ & Hybrid & P: (UCD06OCT) JH & $\begin{array}{l}\text { P: No SETs observed up to a } \\
\text { fluence of } 1 \times 10^{12} \text { with } 63 \mathrm{MeV} \\
\text { protons }\end{array}$ & $\mathrm{N}$ & $5 \mathrm{~V}$ & 3 & $\begin{array}{l}\text { D102506_HCPL6751 } \\
\text { 1111606_HCPL6751 }\end{array}$ \\
\hline \multicolumn{11}{|l|}{ Memories } \\
\hline NAND01GW3B2ANGE & \begin{tabular}{|l|} 
ST \\
Microelectronics
\end{tabular} & 0604 & $\begin{array}{l}1 \mathrm{~Gb} \text { Micro } \\
\text { Flash }\end{array}$ & CMOS & $\begin{array}{l}\text { H: (LBNL07JAN) TO; } \\
\text { P: (IUO6JUL) } \\
\text { TO/MF/RL }\end{array}$ & 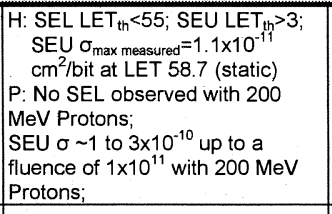 & $\mathrm{N}^{*}$ & $3.3 \mathrm{~V}$ & 1 & $\begin{array}{l}\text { 1072706_NAND01GW3B2ANGE } \\
\text { L012707_NAND01GW3B2ANGE }\end{array}$ \\
\hline K9F4G08U0A & Samsung & 0625 & 4 Gb Flash & CMOS & H: (LBNL07JAN) TO & $\begin{array}{l}\text { SEL LET } T_{\text {th }}>55 \text {; SEU LETth } 3.4 ; \\
\text { SEU } \sigma_{\text {maxmeasured }}=5 \times 10^{-11} \mathrm{~cm}^{2} / \mathrm{bit} \\
\text { (static); } S E U \sigma_{\text {max measured }}= \\
7.5 \times 10^{-11} \mathrm{~cm}^{2} / \mathrm{bit} \text { (dynamic) }\end{array}$ & $\mathrm{N}^{*}$ & $\begin{array}{l}\text { SEL: } 3.3 \mathrm{~V} \\
\text { SEU: } 3.0 \mathrm{~V}\end{array}$ & 3 & L012607_K9F4G08U0A [9] \\
\hline KH41G0X38 & Samsung & 0546 & $\begin{array}{l}1 \text { Gbit DDR } \\
\text { SDRAM }\end{array}$ & $\begin{array}{c}90 \mathrm{~nm} \\
\text { minimum } \\
\text { feature size } \\
\text { CMOS }\end{array}$ & $\begin{array}{l}\text { H: (TAMUO6JUN) RL; } \\
\text { P: (IUO6JUL) RL }\end{array}$ & $\begin{array}{l}\text { H: } \operatorname{SEL} 45<\text { LETth }<108 \\
\text { SEU LET th } ~ \\
\text { P: } \sigma \sim 4 \times 10^{-19} \mathrm{~cm}^{2} / \mathrm{bit} \text { at } 200 \mathrm{MeV}\end{array}$ & $\mathrm{N}^{*}$ & $2.5 \mathrm{~V}$ & 2 & T062806_1072806_KH41G0X38 \\
\hline \multicolumn{11}{|c|}{ Communication Devices } \\
\hline TLK2711 & \begin{tabular}{|l} 
Texas \\
Instrument
\end{tabular} & 0545 & Transceiver & CMOS & H: (LBNLO6SEP) JH & 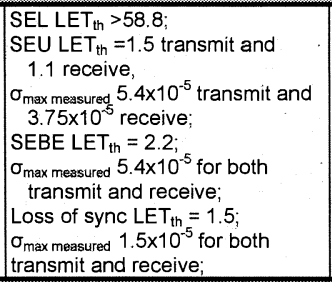 & $\mathrm{Y}$ & $2.5 \mathrm{~V}$ & 2 & L092106_TLK2711 \\
\hline \multicolumn{11}{|l|}{ Power Devices } \\
\hline SHD85202 & Sensitron & 0321 & $\begin{array}{l}\text { Dual power } \\
\text { MOSFET driver }\end{array}$ & $\begin{array}{l}\text { Bipolar/ } \\
\text { CMOS/ } \\
\text { DMOS }\end{array}$ & H: (LBNLO6JUN) JH & $\begin{array}{l}\text { SEL LET } \text { Th }_{\text {th }}>58.8 \text {; SET LETth }<20 \\
\text { at room temp. and }<10 \text { at } 100 \\
\text { degrees Celsius. }\end{array}$ & $\mathrm{Y}$ & $12 \mathrm{~V}$ & 3 & L061706_SHD85202 \\
\hline 53111 & Micropac & 0103 & $\begin{array}{l}\text { Power } \\
\text { MOSFET } \\
\text { Optocouplers }\end{array}$ & Hybrid & $\begin{array}{l}\text { H: (TAMUO6NOV) JH; } \\
\text { P: (UCD06OCT) JH }\end{array}$ & $\begin{array}{l}\text { H: SEL LET } \\
\text { SEGR LET }<83.4 ; \\
\text { No SET testing of of opto due } \\
\text { to device construction } \\
\text { P: No SETs observed up to a } \\
\text { fluence of } 1 \times 10^{12} \text { with } 63 \mathrm{MeV} \\
\text { protons }\end{array}$ & $\mathrm{Y}$ & $\begin{array}{l}\text { OV, } 28 \mathrm{~V}, \\
\text { and } 34 \mathrm{~V}\end{array}$ & $\begin{array}{l}\text { P: } 3 \\
\text { H: } 2\end{array}$ & D102506_T111606_53111 \\
\hline MSAFX11P50A & Microsemi & 0644 & \begin{tabular}{|l} 
Power \\
MOSFET
\end{tabular} & MOSFET & H: (TAMU07FEB) CP & $\begin{array}{l}\text { No SEGR at } O V \text { and }-425 \mathrm{~V} \text { at } \\
\text { LET } 20.6\end{array}$ & $\mathrm{~N}$ & N/A & 10 & T021907_MSAFX11P50A \\
\hline \multicolumn{11}{|l|}{ Special Devices } \\
\hline OMH3075S & $\begin{array}{l}\text { Optek } \\
\text { Technology }\end{array}$ & $\begin{array}{l}\text { SN0011, } \\
\text { SN0137, } \\
\text { and SN0136 }\end{array}$ & $\begin{array}{l}\text { Hallogic Hall } \\
\text { Effect Sensor }\end{array}$ & Bipolar & H: (LBNL06SEP) AS & 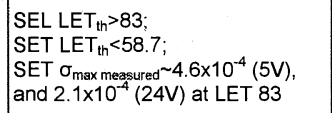 & $\mathrm{Y}$ & $\begin{array}{l}5 \mathrm{~V} \text { and } \\
24 \mathrm{~V}\end{array}$ & 3 & L091906_ОМH3075B \\
\hline TGL4302 & Triquint & $\begin{array}{l}\text { No LDC; } \\
\text { die pkg at } \\
\text { GSFC }\end{array}$ & $\begin{array}{l}\text { MMIC voltage } \\
\text { variable } \\
\text { attenuator }\end{array}$ & $\begin{array}{l}\text { PHEMT } \\
\text { MMIC }\end{array}$ & H: (TAMUO6AUG) SB & $\begin{array}{l}\text { SEL LET } \\
\text { SET }>78.4 \\
\text { SET LET }\end{array}$ & $\mathrm{Y}$ & $10 \mathrm{~V}$ & 1 & T082806_TGL4302 \\
\hline \multicolumn{11}{|l|}{ Discrete Devices } \\
\hline 2N2222 & Microsemi & 0315 & Transistor & Bipolar & H: (LBNLO6JUN) SB & $\begin{array}{l}\text { SEL } \text { LET }_{\text {th }}>58.72 ; \\
\text { SET LET } \\
\text { SET } \sigma_{\text {max }} \sim 20 ; \\
\text { LET } 58.72\end{array}$ & $\mathrm{~N}$ & $8 \mathrm{~V}$ & 1 & L061806_2N2222 \\
\hline
\end{tabular}




\section{TEST RESULTS AND DISCUSSION}

As in our past workshop compendia of GSFC test results, each DUT has a detailed test report available online at http://radhome.gsfc.nasa.gov [8] describing in further detail, test method, SEE conditions/parameters, test results, and graphs of data.

This section contains a summary of testing performed on a selection of featured parts.

\section{1) Agilent Technologies HCPL625K Four Channels Logic Gate Optocoupler}

HCPL625K four-channel logic gate optocoupler was tested for proton-induced SET susceptibility at IUCF. HCPL625K optocoupler has a $5 \mathrm{MHz}$ bandwidth. Previous data [10] shows that fast optocouplers ( $>10 \mathrm{MHz}$ bandwidth) are sensitive to proton-induced SET, but that slow optocouplers (about 400 $\mathrm{KHz}$ bandwidth) are not sensitive to proton-induced SET. In the latter case, optocoupler bandwidth is too low to propagate SETs to the device output. No data were available for optocouplers with bandwidth in between these two ranges. Test data show that HCPL is sensitive to proton-induced SETs. SET cross-section curves are shown in Fig. 1. Generally, we see one type of transient where the phototransistor is in the off state and is turned on. This is the case shown in Fig. 1 where the optocoupler is on and its output is high. In this bias condition, measured cross-section is $1 \times 10^{-7} \mathrm{~cm}^{2} /$ channel. A typical SET when device is on is shown in Fig. 2. HCPL 625K is also susceptible to SET when the phototransistor is on and the device is in the off state (low output). In that case the SET probably occurs in device logic gate output. SET sensitivity is significantly lower in this configuration. Maximum measured cross-section is about $2 \times 10^{-9} \mathrm{~cm}^{2} /$ channel. A typical SET when device is off is shown in Fig. 3. [11]

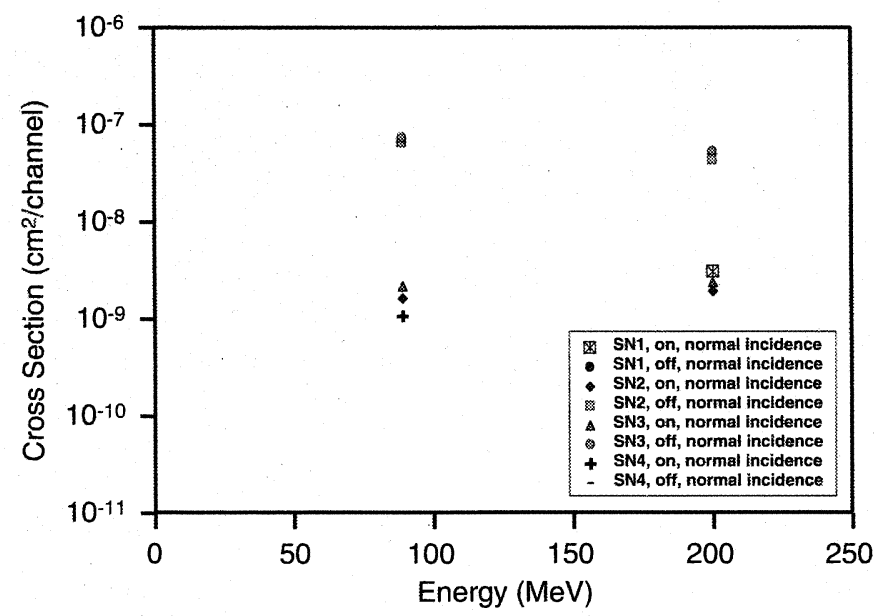

Fig. 1. HCPL625K SET cross-section curves. Cross-section is given for one channel.

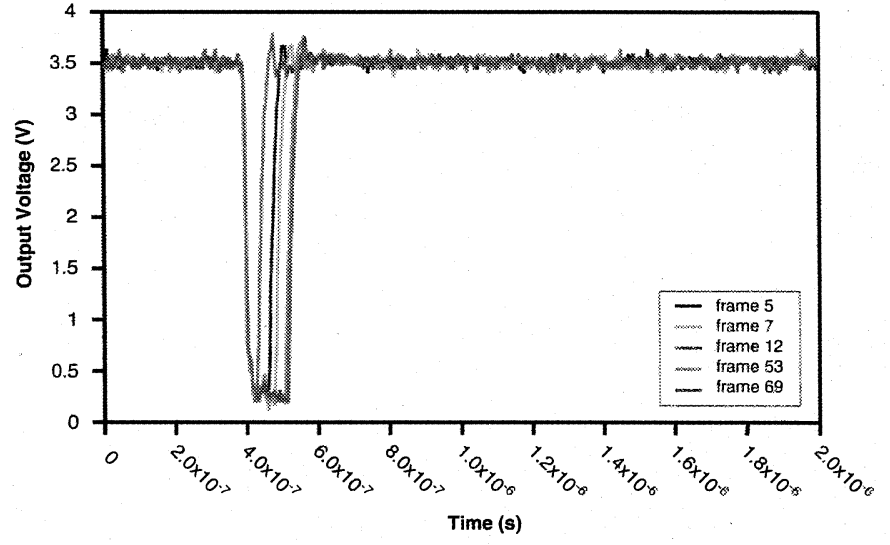

Fig. 2. HCPL625K Typical SET waveforms when optocoupler is on.

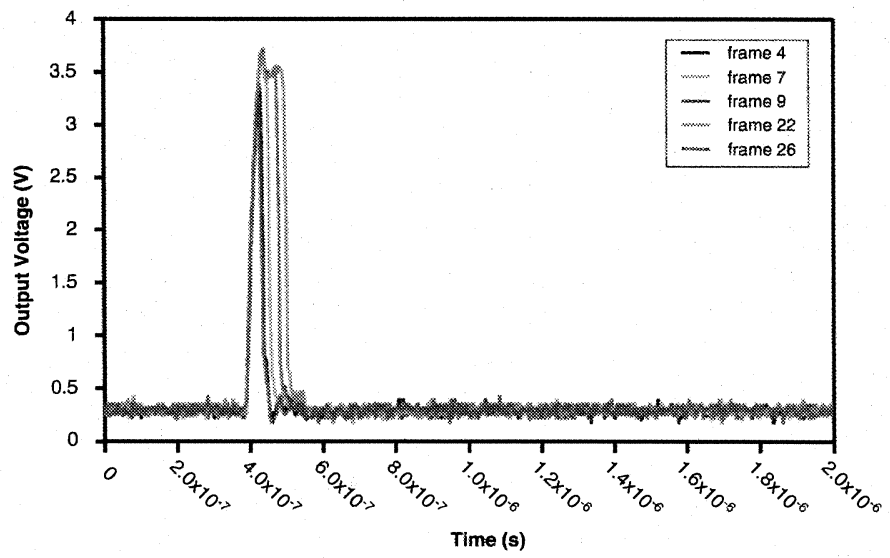

Fig. 3. HCPL625K Typical SET waveform when optocoupler is off.

\section{2) Recent LDO SEE Results}

As with other bipolar analog devices, voltage regulators are known to be sensitive to single event transients (SET). In typical applications large output capacitors are used to provide noise immunity. Therefore, SET amplitude and duration are generally small, and are often of secondary importance. However, even small SETs are a concern for low voltage applications. Over-voltages may cause destructive conditions. Under-voltage may cause functional interrupt and may also trigger electrical latchup conditions. In addition, internal protection circuits which are affected by load as well as internal thermal effects can also be triggered from heavy ions, causing dropouts or shutdown ranging from milliseconds to seconds.

Tested devices are presented in the table to the right. Satcon device is an hybrid developed for the space market. TI devices are commercial parts. All parts have internal protection circuitries for thermal and over current effects.

The parts were tested at LBL with a $10 \mathrm{MeV} / \mathrm{amu}$ beam. Devices under test (DUT) were biased with an input voltage of $3.3 \mathrm{~V}$ and an output voltage of $1.5 \mathrm{~V}$ under different load conditions. Output capacitor values were those recommended in manufacturer data sheets. More detailed results are available in [12].

Figure 4 shows SAT8605R SET cross sections with a 1000 $\mu \mathrm{F}$ output capacitor. We can't see a significant effect of output 
- current on SAT8605R sensitivity. However, SAT8605R is not sensitive for an output current lower than $100 \mathrm{~mA}$.

Only one transient waveform was observed. Figure 5 shows typical transients. Maximum SET amplitude is $450 \mathrm{mV}$. Worst case transient duration is $6 \mu \mathrm{s}$.

Figure 6 shows TPS76701 SET cross sections for a $10 \mu \mathrm{F}$ output capacitor. We can't see a significant effect of output current on device SET sensitivity. However, for high output current values, heavy ions trigger device internal protection circuitries.

Figure 7 shows worst case SET. Device output goes down from $1.5 \mathrm{~V}$ to $0 \mathrm{~V}$. In some cases protection circuitries are triggered and the device output is off for several milliseconds. About $20 \%$ of SET are long duration transients.

Figure 8 shows the SET cross sections for a $10 \mu \mathrm{F}$ output capacitor. SET sensitivity is higher for high output current values. SET sensitivity is negligible for $10 \mathrm{~mA}$ output current. As for TPS76701, for high output currents ( $>100 \mathrm{~mA}$ ), heavy ions trigger device internal protection circuitries.

Figure 9 shows the worst-case transients. Device is shutdown for up to $100 \mathrm{~ms}$. All SET are long duration transients.

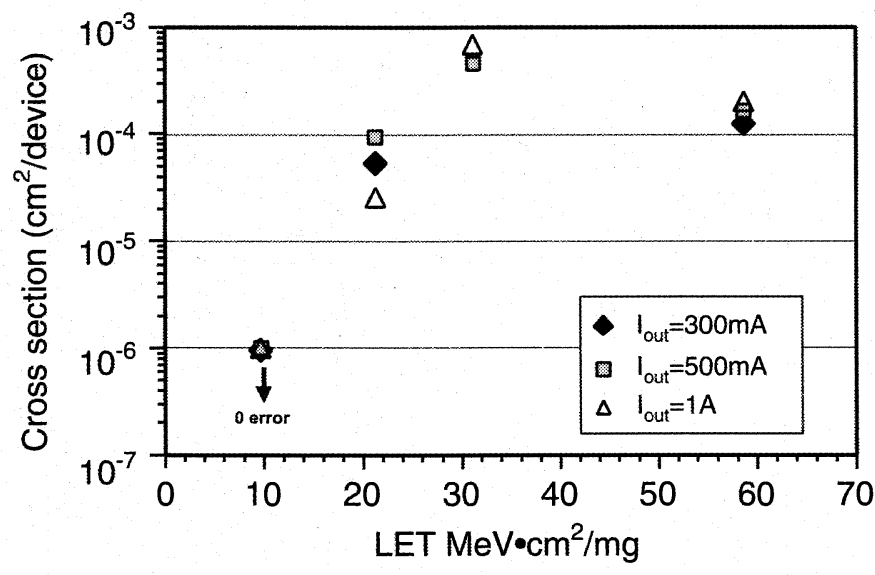

Fig. 4. SAT8605R SET cross-section.

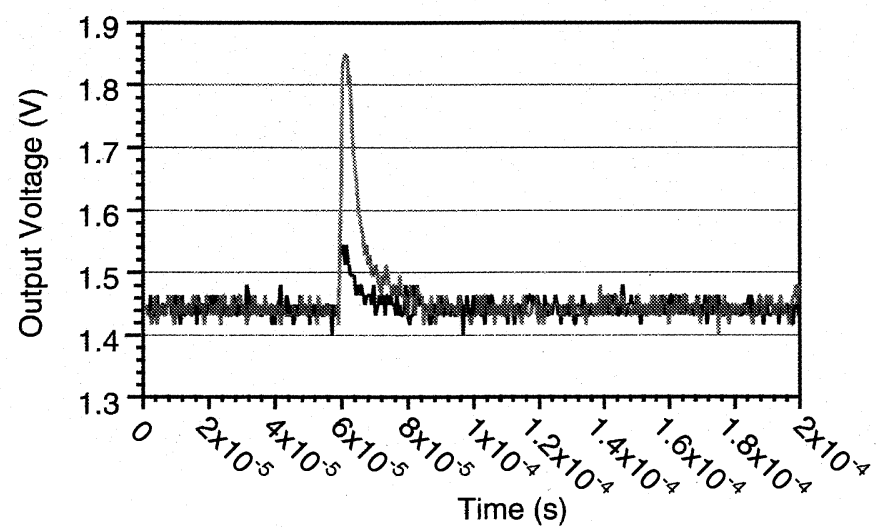

Fig. 5. SAT8605R typical SET.

Fig. 6. TPS76701 SET cross-section.
Fig. 8. TPS73601 SET cross-section curves.

Fig. 9. TPS73601 worst case SETs, $\mathrm{I}_{\text {out }}=250 \mathrm{~mA}$, at $\mathrm{LET}=2.2 \mathrm{MeV} \cdot \mathrm{cm}^{2} / \mathrm{mg}$.

\section{3) Samsung K9F4G08U0A 4 Gb Flash}

The results in Figure 11 were fitted with Weibull parameters, threshold LET $=3.5$, saturation cross section $=5 \times 10^{-}$

${ }^{11} \mathrm{~cm}^{2} / \mathrm{bit}$, width $=27$, exponent $=5$, and Creme 96 was used to calculate the bit error rate for geosynchronous orbit at solar minimum. The result was $1 \times 10^{-12}$ errors/bit-day, which is equivalent to about 1.5 bit errors per year for a 4G. The data in Figure 11, is normalized per device (instead of per bit) so that the SEFI and destructive effects can be shown on the same scale as the bit errors. Obviously, the SEFI and destructive error cross sections are much less than the bit upset cross section, and the error rate expected in space will scale with the cross section.

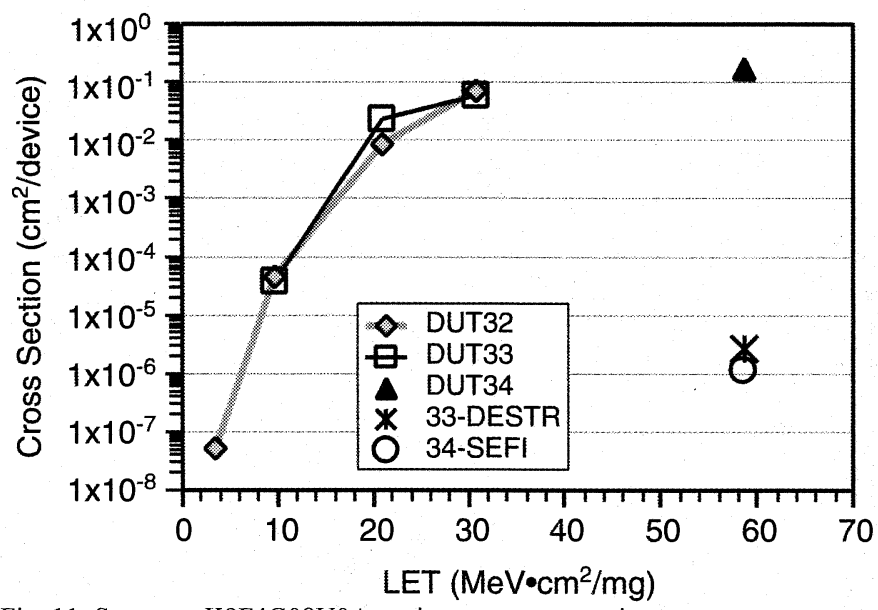

Fig. 11. Samsung K9F4G08U0A static upset cross sections.

4) Microsemi MSAFX11P50A P channel MOSFET

Radiation performance of the $500 \mathrm{~V}, 11 \mathrm{~A}, \mathrm{P}$ channel MOSFET MSAFX11P50A from Microsemi was measured at TAMU. 10 parts were irradiated. 5 out of 10 were irradiated using $1858 \mathrm{MeV}$ Krypton (LET=20.6 $\mathrm{MeVcm}^{2} / \mathrm{mg}$ and Range $=284 \mu \mathrm{m})$. Fig. 10 shows the last passing value and the first failing value on the 5 parts tested with this ion at this energy. Safe operating area is based on this condition.

Fig. 7. TPS76701 SET waveforms, $I_{\text {out }}=1 \mathrm{~A}$, at $\mathrm{LET}=83 \mathrm{MeV} \cdot \mathrm{cm}^{2} / \mathrm{mg}$. 


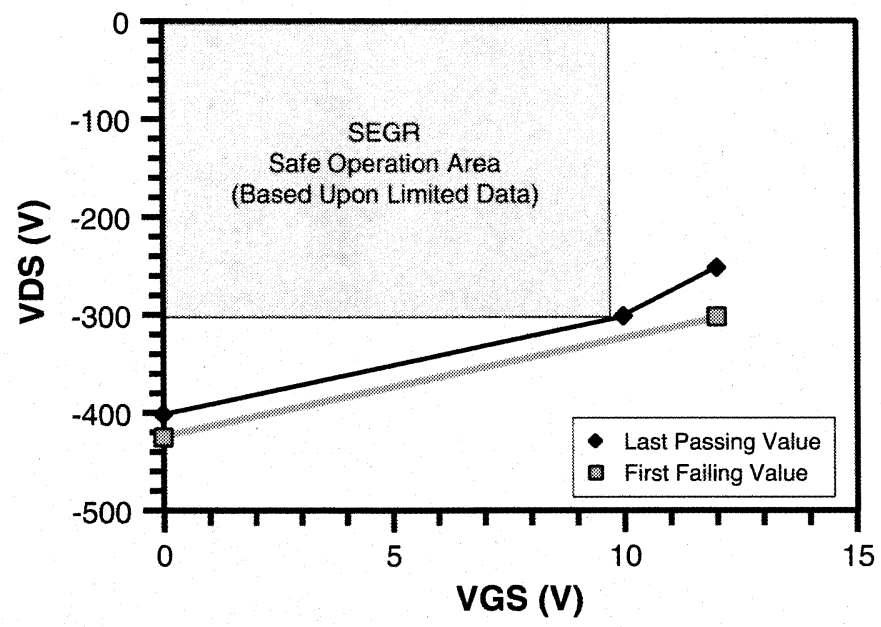

Fig. 10. MSAFX11P50A Krypton test results showing last passing and first failing value. Safe operating area is based upon this condition.

One device was irradiated with $860 \mathrm{MeV}$ Krypton (LET=30 $\mathrm{MeVcm}^{2} / \mathrm{mg}$ and Range $\left.=108 \mu \mathrm{m}\right)$. Three parts were irradiated with $2758 \mathrm{MeV}$ Xenon (LET=40.7 $\mathrm{MeVcm}^{2} / \mathrm{mg}$ and Range $=237 \mu \mathrm{m})$. Two devices were irradiated with $929 \mathrm{MeV}$ Argon $\left(\mathrm{LET}=5.7 \mathrm{MeVcm}^{2} / \mathrm{mg}\right.$ and Range $=445 \mu \mathrm{m}$ ). Table VI shows a summary of all data collected.

Data show that the dominant failure mode is Single Event Gate Rupture (SEGR) and that device operation at $\mathrm{V}_{\mathrm{GS}}<10 \mathrm{~V}$ and $\mathrm{V}_{\mathrm{DS}}<-300 \mathrm{~V}$ provides a relatively safe operating region when irradiated with Krypton and Argon. Krypton represents an upper boundary of concern for space applications. There was a significant decrease in the device operational voltages between $\mathrm{Kr}$ and $\mathrm{Xe}$ with a safe operating region at $\mathrm{V}_{\mathrm{GS}}=-5 \mathrm{~V}$ and $\mathrm{V}_{\mathrm{DS}}<-100 \mathrm{~V}$.[14]

\begin{tabular}{|c|c|c|c|c|c|c|}
\hline $\begin{array}{c}\text { Ion } \\
\text { Spec. }\end{array}$ & $\begin{array}{l}\text { Ion } \\
\text { Energy } \\
(\mathrm{MeV})\end{array}$ & $\begin{array}{l}\text { Serial } \\
\text { Number }\end{array}$ & $\begin{array}{c}\text { Last Passing } \\
\text { Value } \\
\text { (VGS,VDS) }\end{array}$ & $\begin{array}{c}\text { First } \\
\text { Failing Value } \\
\text { (VGS,VDS) }\end{array}$ & \begin{tabular}{|c|} 
SEE \\
Failure \\
Threshold \\
(VGS,VDS) \\
\end{tabular} & $\begin{array}{l}\text { Failing } \\
\text { Nodes }\end{array}$ \\
\hline $\mathrm{Kr}$ & 1858 & 1 & $(0 \mathrm{~V},-425 \mathrm{~V})$ & $(0 \mathrm{~V},-450 \mathrm{~V})$ & $(0 \mathrm{~V},-437 \mathrm{~V})$ & * \\
\hline $\mathrm{Kr}$ & 1858 & 2 & $(10 \mathrm{~V},-300 \mathrm{~V})$ & $(12 \mathrm{~V},-300 \mathrm{~V})$ & $(11 \mathrm{~V},-300 \mathrm{~V})$ & $(G, D)$ \\
\hline $\mathrm{Kr}$ & 1858 & 3 & $(0 \mathrm{~V},-475 \mathrm{~V})$ & $(0 \mathrm{~V},-500 \mathrm{~V})$ & $(0 \mathrm{~V},-488 \mathrm{~V})$ & $(G, D)$ \\
\hline $\mathrm{Kr}$ & 1858 & 4 & $(12 \mathrm{~V},-250 \mathrm{~V})$ & NA & NA & NA \\
\hline $\mathrm{Kr}$ & 1858 & 4 & (0V, $-420 \mathrm{~V})$ & $(0 \mathrm{~V},-440 \mathrm{~V})$ & $(0 \mathrm{~V},-430 \mathrm{~V})$ & (G) \\
\hline $\mathrm{Kr}$ & 1858 & 5 & $(12 \mathrm{~V},-250 \mathrm{~V})$ & NA & NA & NA \\
\hline $\mathrm{Kr}$ & 1858 & 5 & $(0 \mathrm{~V},-420 \mathrm{~V})$ & NA & NA & NA \\
\hline $\mathrm{Kr}$ & 860 & 5 & $(0 \mathrm{~V},-420 \mathrm{~V})$ & NA & NA & NA \\
\hline $\mathrm{Kr}$ & 860 & 5 & $(12 \mathrm{~V},-250 \mathrm{~V})$ & NA & NA & NA \\
\hline $\mathrm{Kr}$ & 860 & 5 & $(0 \mathrm{~V},-480 \mathrm{~V})$ & NA & NA & NA \\
\hline $\mathrm{Kr}$ & 1858 & 5 & $(0 \mathrm{~V},-460 \mathrm{~V})$ & $(0 \mathrm{~V},-480 \mathrm{~V})$ & (0V, $-470 \mathrm{~V})$ & (G) \\
\hline $\mathrm{Xe}$ & 2758 & 6 & $(0 \mathrm{~V},-100 \mathrm{~V})$ & $(0 \mathrm{~V},-125 \mathrm{~V})$ & $(0 \mathrm{~V},-112 \mathrm{~V})$ & \\
\hline $\mathrm{Xe}$ & 2758 & 7 & $(5 \mathrm{~V},-100 \mathrm{~V})$ & $(6 \mathrm{~V},-100 \mathrm{~V})$ & $(5.5 \mathrm{~V},-100 \mathrm{~V})$ & \\
\hline $\mathrm{Xe}$ & 2758 & 8 & $(46 \mathrm{~V}, 0 \mathrm{~V})$ & $(48 \mathrm{~V}, 0 \mathrm{~V})$ & $(47 \mathrm{~V}, 0 \mathrm{~V})$ & (G) \\
\hline $\mathrm{Ar}$ & 929 & 9 & $(0 \mathrm{~V},-480 \mathrm{~V})$ & $(0 \mathrm{~V},-500 \mathrm{~V})$ & $(0 \mathrm{~V},-490 \mathrm{~V})$ & (D) \\
\hline $\mathrm{Ar}$ & 929 & 10 & $(0 \mathrm{~V},-480 \mathrm{~V})$ & $(0 \mathrm{~V},-500 \mathrm{~V})$ & $(0 \mathrm{~V},-490 \mathrm{~V})$ & \\
\hline
\end{tabular}

\section{SUMMARY}

We have presented recent data from SEE tests on a variety of mainly commercial devices. It is the authors' recommendation that this data be used with caution. We also highly recommend that lot testing be performed on any suspect or commercial device. 


\section{ACKNOWLEDGMENT}

The Authors gratefully acknowledge the sponsors of this effort: NASA Electronic Parts and Packaging Program (NEPP), NASA Flight Projects, and the Defense Threat Reduction Agency (DTRA) under IACRO\# 07-4207I. The authors thank members of the Radiation Effects and Analysis Group who contributed to the test results presented here, Cheryl J. Marshall, Paul W. Marshall, Hak S. Kim, Anthony M. Dung-Phan, Donald K. Hawkins, Martin A. Carts, James D. Forney, Timothy L. Irwin, Christina M. Seidleck, Stephen R. Cox, and Mark R. Friendlich.

\section{REFERENCES}

[1] Donna J. Cochran, et al., "Compendium of Current Total Ionizing Dose Results and Displacement Damage Results for Candidate Spacecraft Electronics for NASA," submitted for publication in IEEE NSREC07 Data Workshop, July 2007.

[2] Lawrence Berkeley National Laboratory (LBNL), 88-Inch Cyclotron Accelerator Facility, http//cyclotron.lbl.gov/index.html, May 2007.

[3] B. Hyman, "Texas A\&M University Cyclotron Institute, K500 Superconducting Cyclotron Facility," http://cyclotron.tamu.edu/ facilities.htm, July 2003.

[4] National Superconduction Cyclotron Laboratory (NSCL) at Michigan State University, http://www.nscl.msu.edu/, May 2004.

[5] W.J. Stapor, "Single-Event Effects Qualification," IEEE NSREC95 Short Course, sec. II, pp 1-68, July 1995.

[6] C. M. Castaneda, "Crocker Nuclear Laboratory (CNL) Radiation Effects Measurement and Test Facility," IEEE NSREC01 Data Workshop, pp. 77-81, July 2001.

[7] C. C. Foster, S. L. Casey, P. Miesle, N. Sifri, A. H. Skees, K. M. Murray, "Opportunities for Single Event and Other Radiation Effects Testing and Research at the Indiana University Cyclotron Facility," IEEE NSREC96 Data Workshop, pp. 84-87, July 1996.

[8] NASA/GSFC Radiation Effects and Analysis home page, http://radhome.gsfc.nasa.gov

[9] T.R. Oldham, M. Friendlich, J.W. Howard, M.D. Berg, H.S. Kim, T.L. Irwin, and K.A. LaBel, "TID and SEE Response of an Advanced Samsung 4G NAND Flash Memory," K9F4G08U0A paper to be published in the IEEE NSREC07 Data Workshop, July 2007.

[10] LaBel, Kniffin, et al., "A Compendium of Recent Optocoupler Radiation Test Data," 2000 IEEE Radiation Effects Data Workshop, pg 123-146, July 2000.

[11] Christian Poivey, Anthony Phan, Hak Kim, "SEE Test Report V1.0 Proton test of HCPL625K from Agilent Technologies," http://radhome.gsfc.nasa.gov/radhome/ papers/I032706_HCPL625K.pdf, March 2006.

[12] A. H. Johnston, T. F. Miyahira, F. Irom, and J. S. Laird, "Single Event transients in Voltage Regulators," IEEE Trans. Nuc. Sci., vol 53, no. 6, Dec. 2006.

[13] C. Poivey, A. Sanders, H. Kim, A. Phan, J. Forney, K. LaBel, J. Karsh, S. Pursley, I. Kleyner, and R. Katz, "Single Event Transients in Voltage Regulators for FPGA Power Supply Applications," to be published in RADECS2006 proceedings.

[14] Jeffrey L. Titus, "Test Report (Revision No.1) Final Report Microsemi Power MOSFET (MSAFX11P50A) (Single Event Effects/Survivability)," http://radhome.gsfc.nasa.gov/radhome/ papers/T021907_MSAFX11P50A, Feb. 2007. 


\section{2}

Introduction

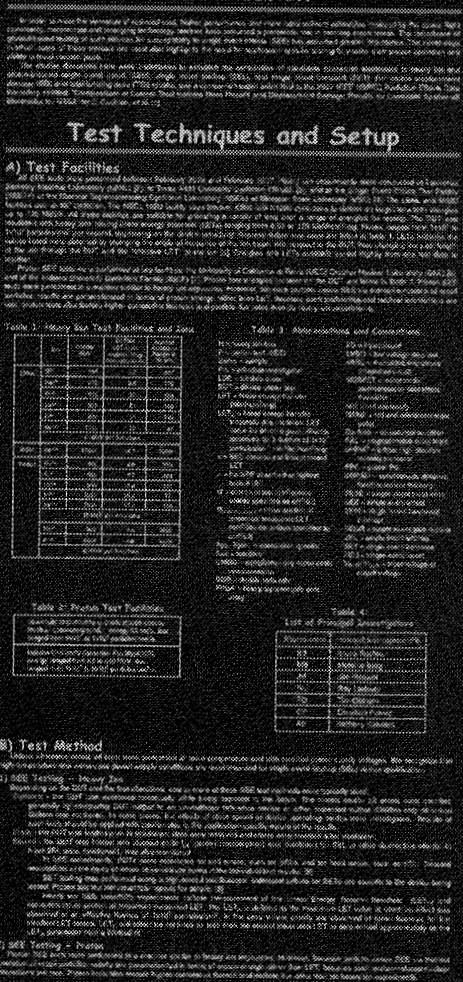

Compendium of Current Single Event Effects Results for Candidate Spacecraft Electronics for NASA

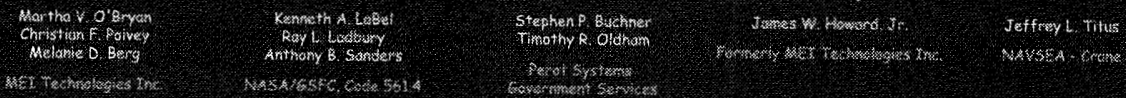

ABSTRACT Sensitivity of a variety of cancioiate spacecraft electronics to proton and heavy ion induced single event efiects is presented.
Devices tested include digital, inear, and hybrid devices.

Sumnary of seE Test Results

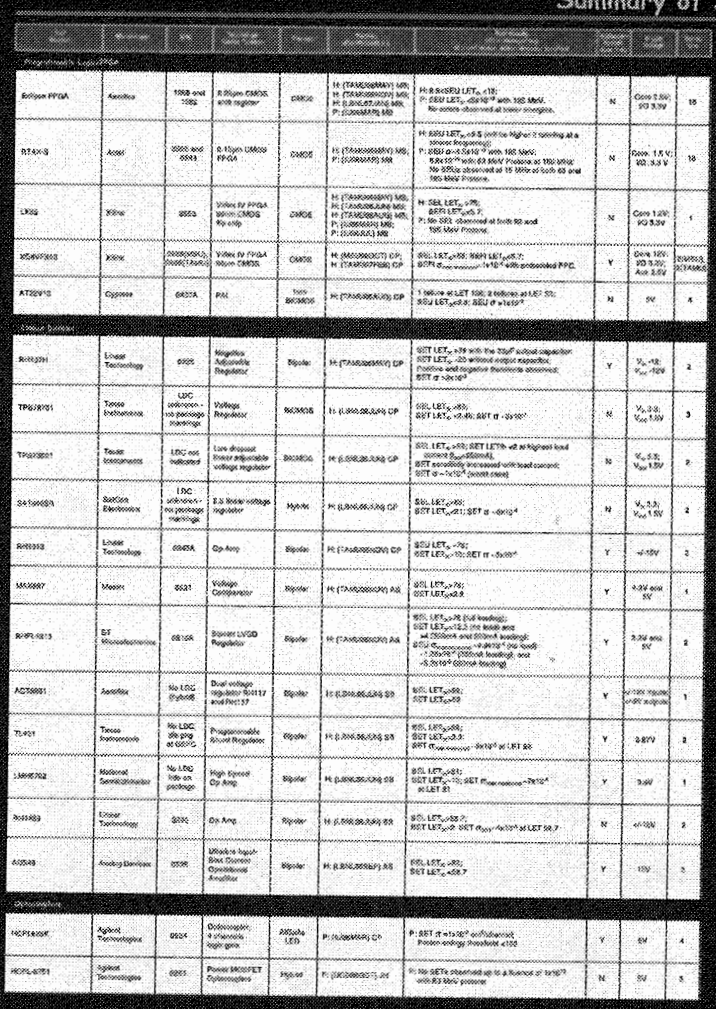

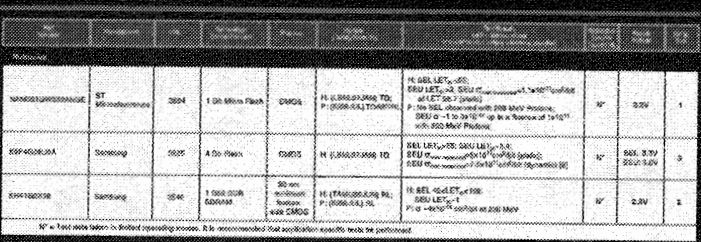

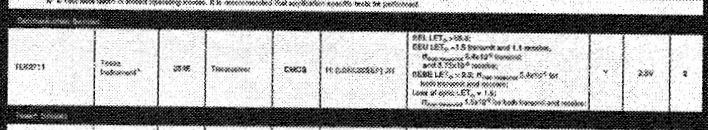

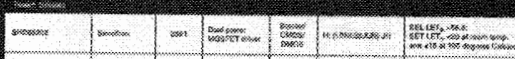

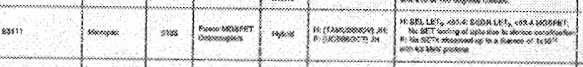

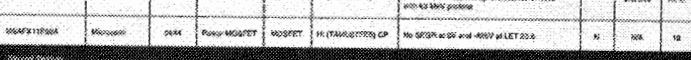

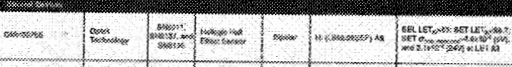

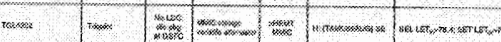

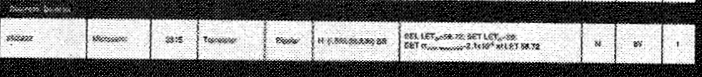
featured Resuits

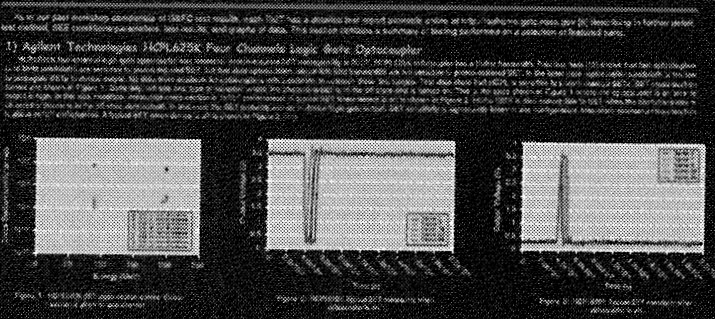

Fearured Results (Cant.)

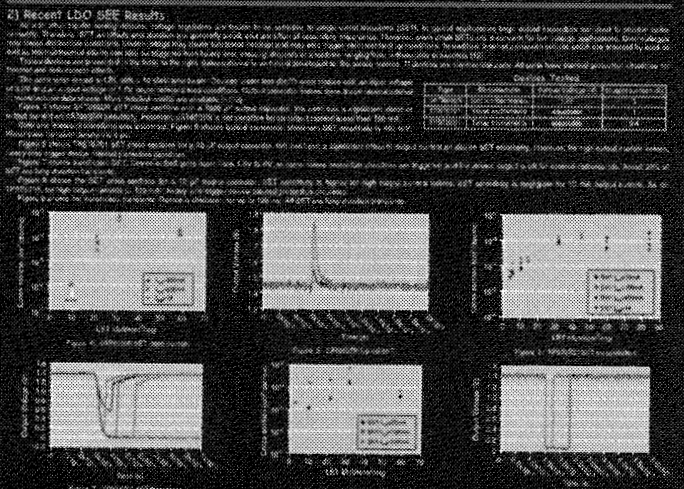

$\cos 2 \sin 20$

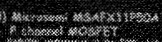

summary

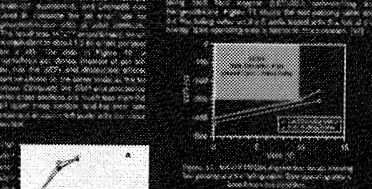
Acknowledanent Acknowecgnent Reterences: i.

Poster for conference containing the same information as the paper. 